\title{
An unpublished CEBPA mutation in familial leukemia
}

\author{
Ayşe Hilal Eroğlu Küçükdiler ${ }^{1}$ (D) - İbrahim Halil Erdoğdu ${ }^{2}$ • Ali Zahit Bolaman ${ }^{1}$ • İrfan Yavaşoğlu ${ }^{1}$
}

Received: 3 September 2020 / Accepted: 28 December 2020 / Published online: 7 January 2021

(C) Springer-Verlag GmbH Germany, part of Springer Nature 2021

A 42-year-old male patient presented with fatigue, fever, and chills. He had no other known disease/s, but his 17-year-old daughter had a history of acute myeloid leukemia (AML). No pathological findings were found on physical examination. Laboratory tests detected pancytopenia. Blasts were observed in the peripheral blood smear. A bone marrow biopsy was performed. The aspirate smear showed that $44 \%$ blasts, some of them containing Auer rods, in the bone marrow. Blasts were positive for MPO and CD34, and negative for CD56 and CD117. CEBPA mutation (c.332_339delCGCCCGCG) was detected from the bone marrow aspiration samples by the next-generation sequencing (NGS) method. We analyzed variants affecting coding areas and \pm 20 splicing regions. Of the target regions, $98.4 \%$ had a coverage depth of more than 250 -fold. It was thought that there may be familial leukemia in the patient who was diagnosed with AML with CEBPA mutation and had a history of AML in his daughter. When the pedigree was performed (Fig. 1), 12 people in the family had AML, and the same CEBPA mutation (c.332_339delCGCCCGCG /p.A111fs*56) was detected in five of these patients. A second CEBPA mutation (c.937_939dupAAG/ p.K313dup) was the same in two of these patients (case-patient and his cousin - person IV.9 in the pedigree). But this mutation was not detected in the other three patients. The other seven patients were not tested, so no results are available. Additional mutations in

Ayșe Hilal Eroğlu Küçükdiler

e_hilal@hotmail.com

1 Faculty of Medicine, Department of Adult Hematology, Adnan Menderes University, Aydın, Turkey

2 Faculty of Medicine, Department of Medical Pathology, Adnan Menderes University, Aydın, Turkey genes other than $C E B P A$ were detected in the AML patients, but no non-CEBPA mutation was detected that was the same in all individuals. Besides, the analysis of two healthy children of the case-patient showed that his healthy daughter had the same CEBPA mutation (c.332_339delCGCCCGCG), while his son does not. The girl who had the mutation but not the disease was 18 years old, and morphological analysis of the marrow was not performed. The girl had the same ASXL1 (c.1934dupG/p.G646fs*12) mutation as her grandmother.

Familial AML with CEBPA mutation represents a recognized leukemia predisposition syndrome, with several families described in the literature. Familial AML associated with germline CEBPA mutation is generally characterized by biallelic $C E B P A$ mutations. The germline mutation occurs in the $\mathrm{N}$-terminal region of $C E B P A$ (as in our family), and a somatic mutation is usually acquired in the C-terminal region at the time of progression to AML. In a study by Tawana et al., 12 separate studies including familial leukemia cases were examined. Ten different germline mutations were identified in these studies [1]. The germline mutation in our case is also different from those described in the literature, and as far as we know, this mutation has not been identified in any familial leukemia case. We present this case to contribute to the literature. 


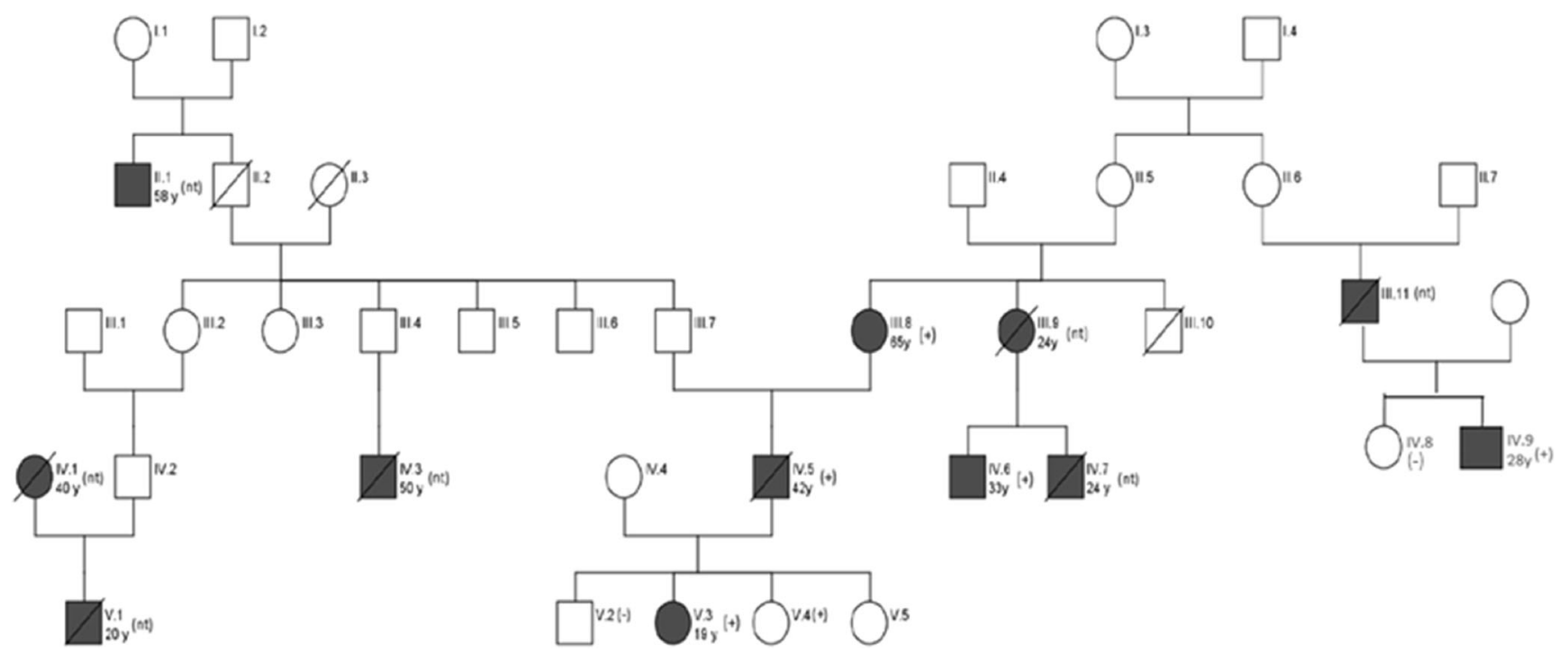

Fig. 1 Pedigree of family. (+): Positive for CEBPA mutation. (-): Negative for CEBPA mutation. (nt): Not tested for CEBPA mutation

Acknowledgments Thanks to doctor Evren Gümüș for giving genetics supervision.

\section{Compliance with ethical standards}

Conflict of interest The authors declare that they have no conflict of interest.

\section{Reference}

1. Tawana K, Rio-Machin A, Preudhomme C, Fitzgibbon J (2017) Familial CEBPA-mutated acute myeloid leukemia. Semin Hematol 54(2):87-93

Publisher's note Springer Nature remains neutral with regard to jurisdictional claims in published maps and institutional affiliations. 